\title{
Current Status and Study Progress of Recombinant Human Endostatin in Cancer Treatment
}

\author{
Kai Li $\cdot$ Mingliang Shi $\cdot$ Shukui Qin
}

To view enhanced content go to www.oncologytherapy-open.com Received: September 12, 2017 / Published online: January 2, 2018

(c) The Author(s) 2017. This article is an open access publication

\section{ABSTRACT}

Angiogenesis plays fundamentally critical roles in solid-tumor pathogenesis, growth, invasion and metastasis. Endostatin, one of the most potent anti-angiogenic factors, was first isolated in Folkman's lab in 1997, and was reported to dramatically shrink tumor blood formation. But its insoluble and unstable nature coupled with the high cost of synthesizing the endostatin protein doomed it for clinical cancer treatment. Intrigued by Folkman's pioneering discoveries, Chinese scientists found a way to refold the protein, making it cost-effective to manufacture a recombinant human endostatin, a soluble and stable form of endostatin. A number of clinical studies have demonstrated the significant

Enhanced content To view enhanced content for this article go to http://www.medengine.com/Redeem/ 1FFCF0600D98ED62.

\section{K. Li}

Department of Thoracic Oncology, Tianjin Key Laboratory of Cancer Prevention and Therapy, Tianjin Medical University Cancer Institute and Hospital, Tianjin, China

M. Shi

The Medical Department, National Key Laboratory for Translational Medicine and Innovative Drugs, Nanjing, China

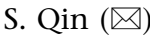

Cancer Center of Bayi Hospital, Nanjing University of Chinese Medicine, Nanjing 210002, China e-mail: qinsk@csco.org.cn survival benefit of rh-endostatin in treating late stage non-small-cell lung carcinoma (NSCLC) and, as a result, rh-endostatin $\left(\right.$ Endostar $\left.^{\circledR}\right)$ was approved by the State Food and Drug Administration of China (CFDA) in September of 2005 as a treatment option for NSCLC. Since then, increasing bodies of clinical data and experience have been obtained from a variety of other different cancers, such as small cell lung cancer, NSCLC in other settings, including malignant serous effusion, melanoma, colon cancer, gastric cancer, breast cancer, nasopharyngeal cancers, and others. This review aims at summarizing current clinical data of rh-endostatin including its survival benefits, optimized dosages, routes of administration, recommended duration and frequency of treatment, predictive biomarkers, and its safety profile in lung cancers as well as other cancers.

Keywords: Adverse events; Angiogenesis; Cancer treatment; Human recombinant endostatin

\section{INTRODUCTION}

Angiogenesis plays fundamentally critical roles in solid-tumor pathogenesis, growth, invasion and metastasis. It is a multi-step process that starts with hypoxia-induced upregulation of proangiogenic factors, such as vascular endothelial 
growth factor (VEGF) and basic fibroblast growth factor (bFGF), which induce the activation of endothelial cells. In turn, these activated endothelial cells secrete matrix metalloproteinases (MMPs), release themselves into the extracellular matrix and degrade the capillary wall, where a branch point in the vessel wall is formed. Next, the free endothelial cells migrate towards the angiogenic stimuli and then re-organize themselves to form tubules with a central lumen. The activated endothelial cells induce the fibroblast cells to produce more extracellular matrix to support the new vessels. Further interconnection of the new tubules follows, forming a branched network of vessels. Similarities in expression have been demonstrated among growth factors secreted by endothelial cells within tumors and their receptors [1].

In recent years, targeted therapies, especially anti-angiogenic therapeutics, have been hot topics in the cancer field. Endostatin, one of the most potent anti-angiogenic factors, was first isolated in Folkman's lab in 1997, and was reported to dramatically shrink tumor blood formation in mice [2], and interfere with multiple key angiogenesis processes. Pre-clinical studies showed that endostatin also inhibited tumor cell growth [3-5], but its insoluble and unstable nature coupled with the high cost of synthesizing the endostatin protein doomed it for clinical use. Intrigued by Folkman's pioneering discoveries, Chinese scientists found a way to refold the protein, making it cost-effective to manufacture a soluble and stable form of endostatin. Recent studies of this recombinant human endostatin (rh-endostatin) demonstrated its ability to down-regulate expression of VEGFs and VEGF receptors (VEGFRs) to inhibit tumor angiogenesis and block VEGF-C signaling to inhibit tumor lymphangiogenesis. Rh-endostatin was also shown to down-regulate other important signaling pathways such as hypoxia inducible factor 1 alpha (HIF-1 $\alpha$ ), MMPs, bFGF, and the integrin $\alpha v \beta 3$ pathway, and thus contribute to inhibition of the tumor micro-environment and restore vascular normalization [7]. These processes are thought to help overcome the physiological barriers to drug and oxygen delivery within tumors, thus enhancing the delivery and antitumor activity of chemotherapy and radiation. A number of clinical studies have demonstrated the significant survival benefit of rh-endostatin in treating late stage non-smallcell lung carcinoma (NSCLC). As a result, rhendostatin (commercial name of Endostar) was approved by the State Food and Drug Administration of China (CFDA) in September of 2005 as a treatment option for NSCLC.

Evidence from clinical studies has shown that rh-endostatin involves the regulation of multiple signaling pathways, targeting wider ranges of signaling molecules, their receptors, growth factors and enzymes, compared to other anti-angiogenic targeted therapies, such as monoclonal antibodies (mAbs) and tyrosine kinase inhibitors (TKIs). It not only inhibits tumor angiogenesis, but also tumor lymphangiogenesis, both of which inhibit tumor growth. Furthermore, rh-endostatin has been demonstrated to induce less drug resistance, and to be associated with less severe toxic profiles, and thus have better patient compliance, which makes the drug more suitable for long term anti-tumor treatment and more effective in preventing tumor recurrence and metastasis compared to other anti-angiogenic targeted therapies. It has been shown that rh-endostatin is safe and effective in squamous cell carcinomas, such as squamous cell carcinomas in lung, where $\mathrm{mAbs}$ and TKIs are not approved due to safety concerns [3-6]. Due to these observations, this review focuses on this promising anti-angiogenic drug, rh-endostatin.

In the most recent decade, an increasing body of evidence has emerged relating to the anticancer properties of rh-endostatin for a number of tumors such as malignant serous effusion, melanoma, and nasopharyngeal cancers. This review aims at summarizing the current status and recommendations for clinical utility of rh-endostatin in different cancers, as well as exploring its administration route and dosage, predictive biomarkers and drug safety management.

\section{Compliance with Ethics Guidelines}

This article is based on previously conducted studies and does not involve any new studies of 
human or animal subjects performed by any of the authors.

\section{CLINICAL UTILITY OF RH- ENDOSTATIN IN CANCER TREATMENT}

\section{Primary Lung Cancer}

Primary lung cancer is one of the most prevalent cancers in China. Based on data from the 2014 National Cancer Registry, primary lung cancer was at the top of the list with 605,900 new cases in 2010 (19.59\% of all new cancer cases), and an incidence of 35.23/100,000. The mortality of primary lung cancer in 2010 was 486,600 (24.87\% of total cancer deaths) [8].

Pathologically, primary lung cancers are divided into two subtypes: small cell lung cancer (SCLC) and non-small cell lung cancer (NSCLC). The majority (80\%) are NSCLC and can be further divided into squamous and nonsquamous subtypes.

\section{Rh-Endostatin in the Treatment of Advanced Stage NSCLC}

Results from the phase III clinical trial led by Sun et al. [9] showed that patients treated with a combination of rh-endostatin and chemotherapy (vinorelbine/cisplatin) exhibited higher objective response rates (ORR) and clinical benefit rates (CBR), defined as the percentage of patients with complete remission, partial remission, or disease stabilization. The combination group also exhibited a longer time to disease progression (TTP) and longer overall survival (OS) with an acceptable safety profile as compared to chemotherapy alone [9]. In detail, the ORR of the combination group $(n=322)$ and the chemotherapy group $(n=164)$ were $35.4 \%$ and $19.5 \%$, respectively $(p=0.0003)$. The median TTP were 6.3 and 3.6 months, respectively $(p<0.001)$; the CBR were $73.3 \%$ and $64.0 \%(p=0.035)$, respectively, and the OS were 13.75 vs. 9.77 months $(p<0.0001)$, respectively. In addition, similar safety profiles were observed between the two groups. In the treatment-naïve population, the OS of the combination and the chemotherapy alone groups were 400 and 278 days, respectively $(p=0.0001)$. Stratified analysis demonstrated that this synergistic effect occurred regardless of pathological subtypes, and was more significant in patients above the age of 60 , indicating the potential of combination of rh-endostatin and chemotherapy in treating older patients with advanced stage NSCLC.

Study results from a phase IV clinical trial with a large study cohort of 2725 NSCLC patients were presented at the 2010 American Society for Clinical Oncology (ASCO) annual meeting [10]. Rh-endostatin was again shown to have synergistic effects with first line platinum combination chemotherapy with an increased OS (median OS of 17.93, and median TTP of 8.26 months in the combination group). Rhendostatin showed comparable and acceptable safety profiles in both adenocarcinoma and squamous cell carcinoma patients based on a stratified analysis. For patients older than 65, addition of rh-endostatin into platinum based chemotherapy showed survival benefit compared to chemotherapy alone.

In 2011, Han et al. published results from their randomized, double-blinded, multi-institutional controlled clinical study, which included 126 advanced stage NSCLC patients treated with chemotherapy (carboplatin/paclitaxel) alone or in combination with rh-endostatin [11]. The median prolonging progression free survival (PFS), median ORR and DCR for the combination group, vs. the chemotherapy alone group, were 7.1 and 6.3 months $(p=0.522), \quad 39.3 \%$ and $23.0 \% \quad(p=0.078)$, $90.2 \%$ and $67.2 \%(p=0.004)$, respectively. The percentage of patients who achieved PFS was significantly higher in the combination group than the chemotherapy alone group [92\% and $77 \%(p=0.027)$, and $80 \%$ and $60 \%(p=0.015)$ at 16 and 24 weeks post treatment]. The safety profile of the combination group was comparable to the chemotherapy alone group, and importantly, no hemoptysis was observed in squamous cell carcinoma patients. 
Li et al. reported the results from a prospective randomized double-blinded clinical study [12]. The study aimed at evaluating combination therapy of rh-endostatin with docetaxel in advanced NSCLC patients who halted first line chemotherapy due to toxicity or progression after first line chemotherapy. The TTP of the combination vs. the chemotherapy alone groups were 2.63 and 2.07 months $(p=0.079)$. Among the patients who achieved stable disease status after two cycles of treatment, the median TTP were 6.23 and 3.27 months $(p=0.040)$, for the combination and the chemotherapy alone groups, respectively. No statistically significant toxicity was observed between the two groups.

It has also been reported that rh-endostatin decreased hydrocephalus in NSCLC metastasized to the brain. Jiang et al. (2013) reported that compared with radiation alone, the combination of rh-endostatin demonstrated decreased hydrocephalus with no increased toxicity. Even though this decrease did not reach statistical significance in the whole cohort, subgroup analysis showed that the decreases were significant in patients with high VEGFR2 expression (93\% vs. $67.7 \%, p=0.012)$, or positive kinase-insert domain-containing receptor expression $(94.4 \%$ vs. $47.3 \%$, $p=0.002$ ) [13].

Metastatic bone disease is a frequent complication of advanced NSCLC. In a recent study by Zhang et al., 33 patients with advanced NSCLC metastasized to bone were randomized to receive vinorelbine/cisplatin (with or without rh-endostatin). The results showed a higher ORR (30\% vs. $0 \%)$, longer OS $(21.44 \pm 17.28$ vs. $7.71 \pm 4.68$ months) in the combination than in the control group $(p<0.05)$ [14].

In summary, these clinical studies demonstrated the efficacy and safety of rh-endostatin in NSCLC, in combination with a variety of chemotherapeutic agents, for both squamous cell carcinoma and adenocarcinoma, and for patients who are chemotherapy naïve or previously treated with chemotherapies. Rh-endostatin has also shown to be effective in decreasing hydrocephalus in NSCLC patients with brain metastasis and may also be effective in treating NSCLC patients with bone metastasis.

\section{Rh-Endostatin in Combination with Chemotherapy or Radiation Therapy in Non-Resectable Stage III NSCLC}

Jiang et al. reported that the combination of rhendostatin with radiation therapy improved short-term and long-term prognosis of patients with NSCLC [15]. Vascular imaging analysis demonstrated that vascular normalization took place 1 week following rh-endostatin treatment, and application of radiation therapy within this window may improve patient response. Similar findings were also reported by Bao et al. [16]: rhendostatin [intravenous injection (IV) at $7.5 \mathrm{mg} / \mathrm{m}^{2} /$ days for 7 days every other week] demonstrated efficacy and a good safety profile in the treatment of locally advanced non-resectable NSCLC (stage III) in combination with radiation therapy and gemcitabine/cisplatin chemotherapy [16].

In order to further evaluate the efficacy and safety of IV rh-endostatin every other week in combination with radiation and chemotherapy in non-resectable stage III NSCLC, a prospective multi-institutional phase II clinical study was conducted in China between 2012 and 2015 [17]. A total of 73 stage III NSCLC patients, including 41 squamous cell carcinomas, 19 adenocarcinomas, 1 large cell carcinomas and 2 undifferentiated carcinomas, were enrolled in the study $($ IIIA $=27$ and IIIB $=36)$. Rh-endostatin was given via IV pump at $7.5 \mathrm{mg} / \mathrm{m}^{2} /$ days for 5 days at week $1,3,5$, and in combination with radiation therapy starting at week 2 for $6-7$ weeks at $60-66 \mathrm{~Gy}$, and with etoposide at $50 \mathrm{mg} / \mathrm{m}^{2}$ on days $1-5$ and cisplatin at $50 \mathrm{mg} / \mathrm{m}^{2}$ on days 1,8 , and 28. The CTCAE3.0 system was used to evaluate side effects and RECIST1.1 was used to evaluate efficacy at 4 weeks post completion of the combination treatment. There were a total of 63 evaluable patients; 61 completed the treatment. ORR at 4 weeks post completion of treatment was $76 \%$. The median PFS was 14.8 months (with a median follow-up time of 13.6 months). PFS and OS at 1 year post-treatment were 51\%, and $78 \%$, respectively. There were 23 cases of level 3 or 4 neutropenia, 9 cases of level 3 or 4 anemia, 10 cases of level 3 or 4 thrombocytopenia, 3 cases of level 3 nausea/vomiting, 8 cases of level 3 radiation esophagitis, and 12 and 2 cases 
of level 1 or $2 / 3$ RP. No cardiovascular toxicity above level 2 was observed.

In summary, these initial clinical studies indicate that IV rh-endostatin treatment in combination with radiation and chemotherapy results in good short term survival and responses, in addition to good safety profiles and patient compliance, in non-resectable stage III NSCLC. This promising treatment warrants further evaluation with larger patient cohorts and longer clinical follow-ups.

\section{Rh-Endostatin in Adjuvant Therapy for NSCLC}

A randomized controlled study including 76 pathologically confirmed NSCLC patients after complete resection surgery compared adjuvant chemotherapy (cisplatin and paclitaxel) alone and in combination with rh-endostatin [18]. The combination group had a longer DFS than the chemotherapy alone group (39.4 vs. 27.6 months, $p<0.05$ ), and a higher 3 year survival rate $(89.4 \%$ vs. $57.9 \%, p<0.05)$.

Another study of 121 stage IIB or IIIA NSCLC patients who had completed resectional surgery showed that 4 weeks of combination adjuvant therapy with rh-endostatin $(n=63)$ decreased disease recurrence at 12 months compared with chemotherapy alone $(n=58)(9.5 \%$ vs. $25.9 \%$, $p<0.05)$, with no statistically significant different side effects $(p>0.05)$ [19]. Similar findings were also observed in stage IIIA NSCLC patients following complete resection, with a DFS of 29.5 vs. 16.0 months $(p=0.015)$ in the combination group vs. the chemotherapy alone group; the 2 treatment groups showed similar toxicity profiles [20].

In summary, the use of rh-endostatin in combination with cisplatin and paclitaxel-based chemotherapy demonstrated encouraging long term survival benefits, as well as a good safety profile, in the adjuvant therapy for NSCLC patients after complete resection surgery.

\section{Rh-Endostatin in the Treatment of SCLC}

Combination therapy of rh-endostatin with chemotherapy (etoposide plus cisplatin) was shown to have promising results in a 2011 single-arm phase II clinical study in patients with extensive SCLC (ED-SCLC) [21]. The median PFS and OS of the 33 ED-SCLC patients enrolled in the study were 5 and 11.5 months, respectively, and the ORR was $69.7 \%$.

Based on these results, a multi-institutional randomized controlled phase II clinical study was conducted, enrolling half of 138 treatmentnaïve ED-SCLC patients in each of two groups: an rh-endostatin and chemotherapy (etoposide plus carboplatin) combination group $(n=69)$ and a chemotherapy (etoposide plus carboplatin) alone group $(n=69)$. The two groups had similar OS and PFS. A statistically significant longer PFS was observed in female patients only (HR $=0.4,95 \%$ CI $0.2-0.9, p=0.020$ ). The ORR of the patients who completed the treatments was significantly higher in the combination group ( $86.7 \%$ vs. $71.0 \%, p=0.046)$, but not in the whole cohort. Patients in the two groups exhibited similar side effects. Quality of life (QOL) score was higher in the combination group at 4 or 6 weeks post treatment [22].

A statistically significant difference in median PFS and ORR were observed in the rh-endostatin plus etoposide plus carboplatin group in another study conducted in late stage SCLC patients (ORR and median PFS were $84.2 \%$ and $50.0 \%$, and 7.9 and 6.1 months, respectively, for the combination group and the chemotherapy alone group) [23]. No difference in drug toxicity profiles were observed between the two groups.

In summary, existing studies of combination therapy of rh-endostatin with etoposide plus cisplatin chemotherapy demonstrated similar safety profiles and improved patient quality of life when compared to chemotherapy alone in SCLC. It also showed a trend in improving PFS and ORR, but not OS. Further investigation is needed in this field.

\section{Malignant Pleural and Peritoneal Effusion}

In a prospective, randomized, multi-institutional, phase III study [24], 324 patients with malignant pleural and peritoneal effusion (MPE) were randomized in a 1:1:1 ratio to 
receive rh-endostatin monotherapy (E), or cisplatin (C), or rh-endostatin combined with cisplatin (E-C), and a further stratification according to patient status with or without intracavitary chemotherapy and serosal effusion location. All three groups received the designated therapeutic agents after thoracentesis or peritoneal centesis drainage. Group E received injection of rh-endostatin, Group $\mathrm{C}$ received cisplatin injection. Group E-C received both agents. The ORRs in groups $\mathrm{E}, \mathrm{C}$ and $\mathrm{E}-\mathrm{C}$ were $48.51 \%, 46.39 \%$ and $63.00 \% \quad(p=0.0373)$, respectively. The ORR of group E-C was higher than those of groups E and C $(p=0.0189)$. Patients with no previous intrapleural or intraperitoneal therapy or systemic chemotherapy, pleural effusion, initial fluid management, adequate drainage, bloody effusion, or non-gastric cancer and female who were treated with rh-endostatin combined with cisplatin, had a higher ORR $(p<0.01)$. The ORRs of patients with bloody effusion, especially bloody pleural effusion in groups $\mathrm{E}$ and $\mathrm{E}-\mathrm{C}$, were significantly higher than that of group $\mathrm{C}$ (group $\mathrm{E}$, $\mathrm{C}$ and $\mathrm{E}-\mathrm{C}$ was $71.05 \%, 45.16 \%$ and $80.00 \%$, respectively; $p<0.01)$. The median TTPs of group $\mathrm{E}$ and group E-C were longer than that of group C ( $p=0.0240$, and 0.0046 . respectively); the median TTPs were $68.87,44.95$, and 69.03 days $(p=0.0121)$, respectively. The percentage of patients with improved quality-of life (QOL) and Karnofsky Performance Status (KPS) scores in group E was significantly higher than those in groups C and E-C $(p<0.05$ or $p<0.01)$. The incidence of adverse events in group E was significantly lower than that in group C $(p=0.0005)$, while no significant difference was found between group $\mathrm{C}$ and group E-C $(p=0.2866)$. Most common adverse events included gastrointestinal reactions, hematologic toxicity, fatigue, pain, liver damage, and fever.

In summary, intrapleural or intraperitoneal injection of rh-endostatin alone or in combination with platinum improves clinical responses, prolongs TTP and improves patient QOL, and is an effective, safe and feasible treatment option for malignant serous effusion, with high patient compliance.

\section{Colorectal Cancer (CRC)}

A large number of studies [25-27] showed that rh-endostatin combined with a chemotherapeutic regimen [XELOX (oxaliplatin plus capecitabine), FOLFOX4 (oxaliplatin plus CF plus 5-Fluorouracil), or FOLFIRI (irinotecan plus CF plus 5-Fluorouracil)] were all well tolerated by and effectively improved the ORR and prolong the PFS of patients with advanced CRC.

A single-center, single-group open phase I clinical trial [28] evaluated the efficacy and safety of rh-endostatin on modified FOLFOX6 (MFOLFOX6) chemotherapeutic regimen for patients with advanced CRC. A total of 21 patients were enrolled in the study. Major adverse effects included leukopenia, neutropenia, anemia, anorexia and constipation. One patient discontinued chemotherapy and two patients discontinued rh-endostatin due to hypersensitivity. One patient experienced premature ventricular beats, and one patient experienced ventricular arrhythmias and ST-T changes seen on EKG.

A meta-analysis [29] also found that rh-endostatin in combination with chemotherapy was effective and safe for advanced CRC patients and suggested that it could be used as a routine treatment regimen for advanced CRC.

A prospectively designed study by Qi et al. [30] enrolled patients with stage IV metastatic CRCs (mCRCs). In the study, the experimental group was treated with rh-endostatin combined with FOLFOX4 chemotherapy; the control group was treated with FOLFOX4 chemotherapy alone. The RR and the disease control rates (DCR) were $53.3 \%$ and $36.7 \%$, and $83.3 \%$ and $73.3 \%$, respectively. The median PFS and OS were 7.3 and 5.3 months, and 11.6 and 9.3 months, respectively, $(p<0.05)$. These findings were confirmed in another clinical study [31]. The main adverse effects were mild (level 1-2) hematologic toxicity, gastrointestinal reaction and neurotoxicity.

These results were also confirmed in a recent study suggesting rh-endostatin in combination with chemotherapy as a first-line treatment option for advanced mCRC. 


\section{Melanoma}

Recently, a multi-institution, double-blinded, randomized controlled phase II clinical trial of rh-endostatin or placebo combined with firstline dacarbazine chemotherapy was conducted in patients with unresectable stage IIIc or IV melanoma [32]. A total of 110 melanoma patients enrolled in the study were randomized at the ratio of $1: 1$ to group A (dacarbazine $250 \mathrm{mg} / \mathrm{m}^{2}$ days $1-5+$ placebo days $1-14$ in a 21 day treatment cycle) and group B (dacarbazine $250 \mathrm{mg} / \mathrm{m}^{2}$ day $1-5+$ rh-endostatin $7.5 \mathrm{mg} / \mathrm{m}^{2}$ day $1-14$ in a 21 day treatment cycle). The ORR was $3.7 \%$ vs. $8.9 \%$, the DCR was $33.3 \%$ vs. $53.6 \%(p=0.051)$, the median response rate was 1.5 vs. 4.5 months (HR: 0.578 ; 95\% CI $0.38-0.89 ; p=0.013)$, and the median OS was 8.0 vs. 12.0 months (HR 0.522; 95\% CI $0.33-0.82 ; p=0.005)$, for the placebo group (group A) and the rh-endostatin (group B), respectively. One-year and 2-year survival rates were $22.5 \%$ vs. $49.7 \%$, and $14.3 \%$ vs. $2.2 .2 \%$, respectively for group A and group B. Further subgroup analysis showed that the rh-endostatin and chemotherapy combination group (group A) demonstrated a $93 \%$ or a $62 \%$ reduction in the risk of death in patients with mucosal melanoma (HR: 0.07, 95\% CI 0.009-0.632), or acral melanoma (HR 0.38, 95\% CI 0.20-0.74).

In 2015, ASCO reported a clinical trial of dacarbazine in combination with rh-endostatin in patients with metastatic melanoma [33]. Twenty patients with metastatic acromegaly melanomas but without c-kit and BRAF mutations were enrolled in the study. All patients were treated with dacarbazine $\left(250 \mathrm{mg} / \mathrm{m}^{2}\right.$ days $1-5)$ in combination with rh-endostatin via infusion pump $\left(2 \mathrm{~mL} / \mathrm{h} ; 7.5 \mathrm{mg} / \mathrm{m}^{2} /\right.$ day or $15 \mathrm{mg} / \mathrm{m}^{2} /$ day $\left.1-14\right)$. Results showed that the treatments were well tolerated by all patients. The expected median PFS was approximately 6 months, which was significantly better than that of rh-endostatin given through the conventional route.

Therefore, rh-endostatin combined with dacarbazine is recommended as first-line treatment option for patients with progressive melanoma. The dosage recommended for rh- endostatin for patients with metastatic acral melanoma is $15 \mathrm{mg} / \mathrm{m}^{2} /$ day via infusion pump in combination with dacarbazine.

\section{Gastric Cancer}

$\mathrm{Xu}$ et al. [34] tested rh-endostatin combined with the SOX regimen (S-1 plus oxaliplatin) in the treatment of advanced gastric cancer. The experimental group $(n=80)$ received rh-endostatin combined with the SOX regimen, whereas the control group $(n=85)$ was treated with the SOX regimen alone. There was no significant difference in ORR between the two groups $(53.8 \%$ vs. $42.4 \%, p=0.188)$, but the difference in DCR was statistically significant ( $85.0 \%$ vs. $72.9 \%, p=0.188)$. PFS was also significantly higher in the combination group than in the control group (15.0 vs. 12.0 months, $p=0.0001)$. The most common adverse events included immunosuppression, gastrointestinal pain and neuropathy with no significant differences between the two groups.

A total of 60 patients with advanced gastric cancer were enrolled in one RCT [35]. Neoadjuvant chemotherapy DCF (docetaxel plus cisplatin plus 5-fluorouracil) in the experimental group was combined with rh-endostatin vs. neoadjuvant chemotherapy alone group. Patients were treated with the chemotherapy for 2 cycles and rested for 4 weeks before surgery. The results showed that the tumor resection rate, complete resection rate and exploratory laparotomy rate were $83.3 \%, 46.7 \%$ and $16.7 \%$, and $93.3 \%, 70.0 \%, 6.7 \%$, respectively, for the control and the experimental groups $(p<0.05)$. No statistically significant differences in the adverse events were observed between the two groups.

A meta-analysis [36] included 10 RCTs with a total of 308 patients treated with chemotherapy alone, and 315 treated with chemotherapy combined with rh-endostatin. Compared with chemotherapy alone, the rate of CR $(9.21 \%$ vs. $3.57 \%, \mathrm{RR}=2.24,95 \% \mathrm{CI} 1.21-4.15, p=0.01)$, the rate of $\mathrm{PR}(46.35 \%$ vs. $31.49 \%, \mathrm{RR}=1.47$, 95\% CI $1.21-1.80, p=0.0001)$, ORR (54.6\% vs. $39.39 \%, \quad \mathrm{RR}=1.39, \quad 95 \% \quad \mathrm{CI} \quad 1.17-1.65$, $p=0.0002)$, and CBR $(81.27 \%$ vs. $69.48 \%$, 
$\mathrm{RR}=1.17,95 \%$ CI $1.06-1.28, p=0.0010)$ were all improved compared with the control group. There were no differences in the percentages of patients who experienced gastrointestinal reaction or myelosuppression between the two groups, but the incidence of adverse cardiac events was higher $(12.36 \%$ vs. $4.19 \%$, $\mathrm{RR}=2.82,95 \%$ CI $1.32-6.00, p=0.007)$ in the rh-endostatin group.

In conclusion, existing evidence supports the recommendation of rh-endostatin in combination with chemotherapy (DCF/SOX regimen) as a first-line neoadjuvant treatment for advanced gastric cancer for regular use. It can improve the rate of tumor resection and complete resection without increasing side effects. Attention needs to be paid to its cardiovascular toxicity.

\section{Nasopharyngeal Carcinoma}

A phase II clinical trial of rh-endostatin combining gemcitabine plus cisplatin (GC) chemotherapy in patients with metastatic nasopharyngeal carcinoma [37] showed a median PFS of 19.4 months in patients with the combination therapy $(95 \%$ CI 13.625.1 months). One-year PFS rate, 1-year survival rate, and ORR were $69.8 \%, 90.2 \%$, and $85.7 \%$ (95\% CI 66.4-95.3), respectively. Fourteen cases achieved complete remission (CR). The most common level $3 / 4$ adverse events included neutropenia and thrombocytopenia.

Twenty-two patients with locally advanced recurrent nasopharyngeal carcinoma (rIII-IVb) were enrolled in a study of rh-endostatin combined with radiochemotherapy [38]. Patients received IMRT treatment, platinum-based adjuvant therapy and IV injection of rh-endostatin at $105 \mathrm{mg} / \mathrm{m}^{2}$, continuously for 14 days, in a 21-day treatment cycle. The median followup time of the study was 13 months. Twenty cases achieved CR, and 2 cases achieved PR. Late-stage radiation damage rate (3/5) was 50\%, and the nasopharyngeal mucosal necrosis rate was $31.8 \%$.

Based on these study results, rh-endostatin combined with chemotherapy can be used as first-line treatment for patients with metastatic nasopharyngeal carcinoma. It is also suitable for locally advanced nasopharyngeal carcinoma treated with platinum-based chemoradiotherapy (IMRT and platinum-based adjuvant therapy), in which it can effectively reduce the incidence of radiation-induced nasopharyngeal mucosal necrosis.

\section{Hepatocarcinoma}

Human recombinant endostatin can act on the VEGF receptor as well as on the kinase domain receptor (KDR), preventing VEGF from binding to endothelial cells, thus blocking the VEGF effect and inducing anti-angiogenesis. In liver cancers, endostatin was found to down-regulate VEGF expression and thus significantly suppress the angiogenesis after transarterial chemoembolization therapy (TACE). A large number of studies $[39,40]$ have shown that rh-endostatin may be an effective treatment option for mid to late stage hepatocarcinomas, with improved patient QOL and a higher response to treatment; a higher percentage of patients converted from alpha-fetoprotein (AFP) - positive status to AFP-negative status, with an acceptable safety profile.

A meta-analysis including 17 RCTs [41] showed that rh-endostatin combined with TACE demonstrated superior ORR and lower VEGF level in the tumor tissues at 3, 7, and 28 days after the TACE procedure in patients with mid to advanced primary hepatocarcinomas. The 1-year survival rate in the rh-endostatin-TACE group was also superior to the TACE alone group. The two groups exhibited similar safety profiles. Because all 17 studies included in the meta-analysis were based on Chinese patients, this conclusion is applicable only for Chinese patients.

\section{Breast Cancer}

Jia et al. found that rh-endostatin inhibited angiogenesis of breast cancer, indirectly leading to tumor regression and dormancy [42]. The study also found that the combination of rhendostatin in breast cancer can potentiate the anti-tumor effect of the neoadjuvant 
chemotherapy, and exacerbate tumor regression. Rh-endostatin combined with GP chemotherapeutic regimen in the treatment of metastatic triple-negative breast cancer was also shown to have synergistic effects, demonstrated by the prolonged median TTP without an increase in the adverse events [43].

$\mathrm{Hu}$ et al. [44] evaluated the efficacy and safety of rh-endostatin combined with taxanebased regimens in patients with HER-2-negative metastatic breast cancer. Among the total of 57 patients, the ORRs of all the patients, or patients who previous received first-line, second-line or third-line chemotherapies, were $68.4 \%, 79.3 \%$, $54.5 \%$, and $16.7 \%$, respectively. The median PFS of all patients was 10.8 months, but the median OS was not reached. Median PFS for patients who previously received first, second, or third line treatment were $11.9,7.5$ and 7.4 months $(p=0.048)$, respectively. There was no statistically significant difference in the PFS between hormone receptor positive and negative patients. The most common level $3 / 4$ hematologic toxicities were neutropenia $(80.7 \%)$ and leucopenia $(77.2 \%)$. Six cases $(10.5 \%)$ of feverassociated neutropenia were observed (10.5\%). The most common level 3/4 non-hematologic toxicities included liver damage $(10.5 \%)$ and peripheral neurotoxicity (8.8\%). No deaths occurred in this study.

Therefore, rh-endostatin combined with chemotherapy should be actively considered for advanced stage breast cancer, especially HER2negative metastatic breast cancer, in which for combination of rh-endostatin with paclitaxelbased chemotherapy was demonstrated to be safe and effective, and especially for those changed from HER2 positive to HER2 negative after treatment with first-line chemotherapy. But its long-term efficacy and adverse reactions need to be further confirmed.

\section{Esophageal Cancer}

The literature published in the British Journal of Radiology in 2012 [45] confirmed the value of anti-angiogenic therapy in esophageal cancer. Of the 38 patients with locally advanced unresectable esophageal squamous cell carcinomas enrolled in the study, 18 received rh-endostatin in combination with radiotherapy and chemotherapy (fluorouracil + cisplatin), 20 patients received radiotherapy and chemotherapy only. Significantly more patients achieved $\mathrm{CR}$ in the rh-endostatin combination group (44\% vs $30 \%)$. Longer 1-year survival (72\% vs. $50 \%$ ), 3-year survival (32\% vs. $22 \%$ ), and PFS (11. 3 vs. 8.1 months) were also observed in the rh-endostatin combination group with no increases in adverse effects.

A randomized phase II study [46] recently presented at the 2015 ASCO annual meeting evaluated the efficacy and safety of rh-endostatin combined with CRT in locally advanced esophageal squamous cell carcinoma (SCC). Sixty-three patients were randomly divided to receive treatment of docetaxel $\left[75 \mathrm{mg} / \mathrm{m}^{2}\right.$, day 1)/cisplatin $\left(25 \mathrm{mg} / \mathrm{m}^{2}\right.$, day $\left.1-3\right)$ and IMRT at 60-66 Gy/30-33 times/6-7 weeks] alone or in combination with rh-endostatin. CT perfusion imaging was performed before and after treatment to evaluate tumor microvessel density (MVD). The results showed that the ORR was significantly higher in the rh-endostatin group than in the control group (62.3\% vs. $55.1 \%)$, as well as the 1- and 2-year OS rates $(78.1 \%$ vs. $67.7 \%$, and $56.2 \%$ vs. $45.1 \%$ ). Median PFS was 16.5 vs. 9.3 months $(p<0.05)$. Compared with CRT alone, rh-endostatin combination significantly reduced the tumor blood flow, blood volume and MVD $(p<0.05)$. There was no significant difference in the adverse reactions between the two groups, and no new adverse events related to rh-endostatin were observed.

In summary, chemotherapy combined with rh-endostatin therapy is an alternative treatment for locally advanced esophageal cancer, for which patients are typically faced with poor long-term survival.

\section{Biliary Tumors}

A clinical trial of the XELOX regimen (capecitabine plus oxaliplatin) in combination with rhendostatin as the first-line treatment of patients with advanced biliary tumors [47] was conducted. Forty-two patients with stage IV cholangiocarcinoma, were randomly assigned 
to a combination $(n=18)$ or a chemotherapy alone group $(n=24)$. The chemotherapy group was treated with the XELOX regimen; the combination group included rh-endostatin. The results showed that the median PFS (7.5 vs. 5 months), median OS (14 vs. 9.5 months) and improvements in QOL (77.8\% vs. 66.7\%) in the rh-endostatin combination group were significantly better than those of the control group $(p<0.05)$. The most common adverse reactions were gastrointestinal reactions, hand-foot syndrome, myelosuppression, neurotoxicity and oral mucositis. There was no significant difference in adverse events between the two groups.

A clinical trial of the GEMOX regimen (gemcitabine plus oxaliplatin) in combination with rh-endostatin as first line treatment for advanced biliary neoplasms [48] included 48 patients with stage IVB cholangiocarcinoma. The patients were divided into a chemotherapy alone group $(n=28)$ and a combination of chemotherapy with rh-endostatin group $(n=20)$. Results showed that the combination group had an RR of $20.0 \%$ and a DCR of $80.0 \%$. The median OS was 14.0 months, the QOL improvement rate was $80.0 \%$, the RR was $21.5 \%$ in the chemotherapy alone group and the DCR was $75.0 \%$, the median TTP was 6.0 months and the median OS was 10.0 months. The improvement rate of QOL was $71.4 \%$. There was a significant difference in median TTP and OS between the two groups $(p<0.05)$. The most common adverse events were bone marrow suppression, though most were mild (grade 1-2), and the difference was not statistically significant between the two patient groups $(p>0.05)$.

In conclusion, rh-endostatin in combination with the XELOX or GEMOX regimens may be used as the first-line regimen for patients with advanced (stage IV/IVB) cholangiocarcinoma. However, further studies are warranted.

\section{Epithelial Ovarian Carcinoma (EOC)}

A study was undertaken to investigate the effect of gemcitabine combined with rh-endostatin on patients with recurrent platinum-sensitive epithelial ovarian cancer. The DCR and PFS of the rh-endostatin group were significantly higher compared to the control group $(70.9 \%$ vs. $40.7 \%$, and 6.3 vs. 3.2 months) $(p<0.01)$. The OS was 12.5 months, which was higher than that of the control group (10.4 months) with no statistical significance between the two groups $(p>0.05)$. There was also no significant difference in the adverse events between the two groups [49].

In a recent study [50], enrolled EOC patients were treated with TP regimen (cisplatin, $75 \mathrm{mg}$ / $\mathrm{m}^{2}$, days $1-4$, paclitaxel, $175 \mathrm{mg} / \mathrm{m}^{2}$ ) in combination with rh-endostatin. Administration was via continuous infusion with a portable IV pump in the experimental group, and/or infusion without the pump in the control group. The RR and DCR were $37.5 \%$ and $62.5 \%$, respectively, after two treatment cycles, and $50.0 \%$ and $81.3 \%$, respectively, after four treatment cycles $(p>0.05)$. However, VEGF expression levels and the incidence of adverse events (including myelosuppression and adverse cardiac reactions) were significantly lower in the combination group than in the control group $(p<0.05)$.

In summary, continuous IV infusion is the most widely used and most effective injection method for rh-endostatin administration. It is recommended to use gemcitabine plus rh-endostatin in patients with recurrent platinum sensitive epithelial ovarian cancer.

\section{Cervical Cancer}

Ke et al. [51, 52] investigated the short-term efficacy of rh-endostatin combined with radiotherapy and chemotherapy in the treatment of patients with advanced cervical cancer. They found that rh-endostatin combination therapy improved the short-term clinical efficacy compared with the control group, without increasing the incidence of adverse events. Nong et al. used IV infusion of rh-endostatin combined with gemcitabine in the treatment of advanced cervical cancer in their study [53]. The results showed significantly prolonged PFS in the combination group compared with the gemcitabine alone group (4.4 and 2.9 months, respectively, $p=0.002$ ). The ORR and the 
incidence of adverse reactions (mainly anemia, leukopenia, neutrophils, neutropenia and thrombocytopenia, skin rashes, vomiting, anorexia, constipation, high bilirubin, and thrombosis) in the two groups were not significantly different $(p>0.05)$.

In summary, rh-endostatin combined with gemcitabine or radiotherapy is recommended for patients with advanced cervical cancer.

\section{Osteosarcoma}

Many pre-clinical trials have confirmed that endostatin can inhibit the growth of osteosarcoma cells. Osteosarcoma itself also expresses higher levels of endogenous endostatin, and can be used as a diagnostic marker for osteosarcoma. Patients with lung metastases expressed significantly lower levels of endogenous endostatin, suggesting that endostatin may inhibit osteosarcoma metastasis.

A study of 116 patients with newly diagnosed osteosarcoma [54] showed that rh-endostatin treatment significantly inhibited VEGF expression and microvascularization, significantly prolonged event-free survival and reduced the occurrence of metastasis, suggesting the anti-metastatic potential of rh-endostatin. Once metastasized, the prognosis of osteosarcoma is poor. A prospective non-randomized clinical study [55] explored the longterm efficacy and safety of a combination of chemotherapy with rh-endostatin in the treatment of stage IIB osteosarcoma. Three-hundred and thirty patients were included in the analysis, including 58 patients in the combination treatment group and 272 patients in the chemotherapy only group. In the control group, the survival rates without distant metastasis at 1, 2 or 3 years were $79 \%, 70 \%$ and $65 \%$, respectively, and $93 \%, 86 \%$ and $77 \%$ in the combination group $(p=0.045)$. PFS rates for 1,2 or 3 years were $76 \%, 66 \%$ and $60 \%$, respectively, in the control group, and $90 \%$, $83 \%$ and $74 \%$ in the combined group, respectively $(p=0.025)$. There was no significant difference in OS between the two groups. Cox multivariate analysis showed that the combination of anti-angiogenic therapy reduced the risk of distant metastasis to half that of chemotherapy alone $(\mathrm{RR}=0.46)$. The adverse events in both groups were primarily mild (grade $1 / 2$ ) and there were no significant differences in adverse events between the two groups.

In another study of rh-endostatin combined with neoadjuvant chemotherapy in stage II classic osteosarcoma, 67 patients were divided into neoadjuvant chemotherapy with rh-endostatin $(n=22)$ and chemotherapy alone $(n=45)$ [56]. Chemotherapy regimens included adriamycin, cisplatin, methotrexate, and ifosfamide. The mean follow-up time was 11.5 months. There was no significant difference in tumor necrosis rates between the two groups, but VEGF expression and MVD were significantly lower after surgery in the combination group than the control group. Although rh-endostatin combined with neoadjuvant chemotherapy did not improve the tumor necrosis rate, it was effective in inhibiting tumor neovascularization.

Therefore, anti-angiogenesis therapy has a protective effect in the process of lung metastasis in osteosarcoma. For patients with bone and soft tissue sarcoma metastasized to lung, rhendostatin combined with chemotherapy is recommended.

\section{Soft Tissue Sarcoma}

Zhang et al. [57] explored the clinical efficacy of rh-endostatin combined with chemotherapy in the treatment of advanced soft-tissue sarcoma. The control group received conventional chemotherapy. The experiment group received rh-endostatin combined with chemotherapy. The results showed no significant difference in ORR, but the DCR of the control group was significantly higher than that of the experiment group. The PFS and OS of the experiment group were 120 and 452 days, respectively, which were significantly longer than those of the control group of 70 and 286 days. The 1- and 2-year survival rates of the experiment group were $56.2 \%$ and $30.2 \%$, significantly higher than $35.4 \%$ and $26.5 \%$ in the control group. No serious adverse events were observed in the two 
groups. Huang et al. [58] also found that rhendostatin combined with chemotherapy in the treatment of metastatic soft tissue sarcoma has significant anti-tumor activity, and was well tolerated and worthy of wider clinical usage.

A recent study included 47 patients diagnosed with stage IV bone and soft tissue sarcomas treated with chemotherapy $(n=24)$ or chemotherapy combined with rh-endostatin $(n=23)$. In the combined group and control groups, the median PFS (8.6 vs. 4.4 months) and the clinical benefit response (CBR) $(47.8 \%$ vs. $16.7 \%)$ both showed statistically significant difference ( $p=0.032)$, while the median overall survival (11.7 vs. 10.6 months, $p=0.658)$ and the ORR $(17.4 \%$ vs. $8.3 \%, p=0.167)$ showed no significant difference. The common grade $3 / 4$ side effects for both groups were myelosuppression and transient elevation of transaminases [59].

These results suggested that rh-endostatin combined with chemotherapy may be effective with tolerable adverse effects in the treatment of patients with advanced uterine leiomyosarcoma, advanced or metastatic soft tissue sarcoma, or refractory ESR.

\section{Glioma}

In a study of rh-endostatin combined with temozolomide (TMZ) in the treatment of recurrent high-grade gliomas, 74 patients were randomized to treatment with TMZ alone and rh-endostatin combined with TMZ. The DCR of the TMZ group was $35.1 \%$, the median PFS was 4 months and the PFS rate was $27.0 \%$ at 6 months. The DCR of the combination group was $62.1 \%$ and the median PFS was 6 months, and the 6-month PFS rate was 43.0\%. Compared with the TMZ group, the incidence of hypertension was higher in the combination group. Compared with TMZ chemotherapy alone, the combination of rh-endostatin treatment demonstrated objective efficacy in recurrent high-grade glioma patients with, PFS with a good safety profile [60].

Eleven patients with recurrent glioblastoma multiforme (GBM) were included in the study by Zhang et al. [61]. All of the patients received a combination therapy of rh-endostatin and chemotherapy. Chemotherapeutic agents used for three patients were irinotecan (CPT11), and the chemotherapeutic agents for the other 8 patients were irinotecan and TMZ. Among the 11 patients, 4 patients achieved PR, 3 patients had SD and 4 had PD. The median PFS was 5.5 months, the PFS was $36 \%$, and the median OS was 7.1 months. Adverse reactions were mainly reversible grade $3(9 \%)$ and $4(18 \%)$ neutropenia, with no serious adverse events.

In summary, rh-endostatin combined with irinotecan and TMZ may be used as salvage chemotherapy for recurrent GBM in patients with high grade glioma. However, further study is still needed.

\section{NF2 Schwannoma}

On the basis of results from other pilot studies, Liu et al.(unpublished results) explored the use of rh-endostatin monotherapy in the treatment of NF2 schwannoma (NF2). This ongoing clinical study (NCT02104323) selected 20 NF2 patients with bilateral acoustic neuroma with the risk of hearing loss. Rh-endostatin was administered through continuous IV pump at the dosage of $7.5 \mathrm{mg} / \mathrm{m}^{2} /$ day continuously for 3 months. After 1 month of intermission, treatments were repeated for a total of 3 cycles. All cases were treated for 1 year, and followed up for 6 months. The results showed that $90 \%$ of the patients had different degrees of benefit. Results showed that $17.5 \%$ of the patients had decreased tumor volume by $\geq 20 \%, 25 \%$ of patients achieved $\mathrm{PR}$, and $47.5 \%$ patients achieved SD. Hearing was improved in $23.5 \%$ patients, dizziness decreased $(72.7 \%, 8 / 11)$, mobility was improved $(66.7 \%, 4 / 6)$, local pain was reduced $(75 \%, 3 / 4)$, and nausea and vomiting improved $(100 \%, 3 / 3)$. The main adverse effects included venous pump wound infection, menstrual disorders, and band skin allergies. Symptomatic treatments were sufficient to relieve these discomforts. There were no treatment-related deaths.

Therefore, rh-endostatin as a single agent treatment may be considered for NF2 bilateral vestibular schwannoma as it may inhibit tumor 
growth, prevent hearing loss, and improve clinical symptoms. Long-term continuous use of rh-endostatin showed no significant adverse reactions, and avoided the fluctuation of drug concentration caused by repeated single dosages, while not increasing drug toxicity.

\section{OPTIMIZATION OF RH- ENDOSTATIN TREATMENT STRATEGIES}

\section{Exploration of Treatment Cycle and Duration}

Compelling evidence in the literature suggests that anti-angiogenic treatments should follow strategies that allow patients exposure to all effective drugs through multiple cycles and long courses of treatment.

Li showed in a study of NSCLC that the median TTP was 6 months and the median OS was 18 months for patients who received fewer cycles of rh-endostatin ( $<4$ cycles), compared with the median TTP of 17 months [62]. Conversely, a median OS of 26 months was reported for patients who had received more cycles ( $\geq 4$ cycles) of rh-endostatin treatment. There was no significant difference in adverse events of grade $3 / 4$ among patients with different cycles of treatment, suggesting that rh-endostatin combined with chemotherapy had a long-term effect on patients with NSCLC. Patients experiencing multi-cycle therapy could see significantly improved long-term efficacy and prolonged survival without an increase in adverse effects. In order to more objectively evaluate the importance of the full cycle long course strategy, the researchers retrospectively analyzed 272 cases of advanced NSCLC patients treated with first-line chemotherapy combined with rh-endostatin therapy [63]. Studies with PD after 4 cycles of treatment were excluded from the analysis. The results showed that patients exposed to full cycle long course treatment ( $>4$ cycles) exhibited significantly longer OS than patients who were exposed to shorter courses (23.1 vs. 14.0 months, $p<0.001)$. Stratified analyses demonstrated that the survival benefit was significant regardless of pathological subtype or epidermal growth factor receptor (EGFR) status, with an overall OS of much longer than 1 year, and OS for patients with wild type EGFR of 29.6 months, which matched the OS for patients with EGFR mutations treated with TKIs [9].

Therefore, rh-endostatin combined with chemotherapy can improve short-term efficacy. Long-term efficacy can be improved by following the multi-cycle long course strategy $(\geq 4$ cycles) without increasing adverse events.

\section{Exploration of Administration Route and Dosage}

Rh-endostatin administered at the traditional dosages has been shown to primarily improve DCR and prolong survival. This traditional dosage has been found to be associated with limited improvements in ORR. Therefore, there is a clinical need to identify an administration route and dosage levels to improve ORR and thus boost patient confidence and compliance while maintaining the long-term survival benefit.

The traditional dosage of rh-endostatin is $7.5 \mathrm{mg} / \mathrm{m}^{2}$, administered by IV infusion over a period of $2.5-4 \mathrm{~h}$, using a continuous infusion for 14 days. Work from the Folkman lab suggested that low-dose continuous administration may improve the prognosis of cancer patients treated with broad-spectrum anti-angiogenic drugs, such as rh-endostatin. The pre-clinical stability test confirmed the stability of rh-endostatin for 7 days in the chemo-pump at $37^{\circ} \mathrm{C}$. Phase I clinical trials [28] showed that continuous infusion of rh-endostatin $\left(7.5-30 \mathrm{mg} / \mathrm{m}^{2}\right)$ for 28 days was safe and the maximum tolerance dose (MTD) and dose-limiting toxicity (DLT) were not observed. Hansma et al. [64] further confirmed the safety of large dosages of rh-endostatin in human experiments, using a longest continuous IV infusion of 28 days and a maximum dose of subcutaneous injection of $120 \mathrm{mg} / \mathrm{m}^{2}$.

Another phase I clinical trial of rh-endostatin combined with pemetrexed plus carboplatin in the treatment of advanced NSCLC [65] showed 
that continuous IV infusion of rh-endostatin at $7.5-30 \mathrm{mg} / \mathrm{m}^{2}$ per day for 20 days demonstrated stable plasma concentration, and was well tolerated by patients; this treatment did not increase the incidence of chemotherapy-related adverse events. Furthermore, no adverse reactions were found to be correlated with the dosages of rh-endostatin. The best short-term efficacy was observed at a dose of $15 \mathrm{mg} / \mathrm{m}^{2}$ for 4 cycles with DCR of $94.1 \%$, and ORR of $76.5 \%$.

In summary, continuous IV administration of rh-endostatin via infusion pump is widely accepted clinically due to its convenience and good patient compliance, and its effectiveness in maintaining stable plasma concentration. Two multi-institutional clinical trials of continuous IV infusion of rh-endostatin at the higher dose of $15 \mathrm{mg} / \mathrm{m}^{2}$ in combination with chemotherapy in the treatment of advanced lung squamous cell carcinoma are underway (NCT02513342 and NCT02283476), both of which are expected to observe significant increases in ORR.

\section{Exploration of Drug Administration Sequence and Timing}

Ning et al. [66] established a lung cancer nude mice model to study the window of vascular normalization. Using this model, vascular normalization was found 3-6 days after rh-endostatin treatment and administration of paclitaxel in this window was the most ideal. Through their studies of Lewis lung cancer, Huang et al. [67] suggested that rh-endostatin was likely to normalize tumor vascularity and microenvironment through modulation of the balance between VEGF-A and TSP-1.

Li et al. [68] reported that rh-endostatin may modulate anti-angiogenic factors and temporarily normalize tumor vessels. Peng et al. [69] also showed that rh-endostatin induced tumor vascular normalization, reduced hypoxia in nasopharyngeal carcinoma and significantly enhanced sensitivity to radiotherapy. Results from these murine models were important in supporting the use of rh-endostatin combined with radiotherapy in the treatment of nasalpharyngeal cancer.
CT perfusion imaging and hypoxic imaging were used in assessing the dynamic changes in blood perfusion and hypoxic states in NSCLC patients treated with rh-endostatin [70]. This study revealed that the permeability surface (PS) and the hypoxia of the tumor tissues $(T / N)$ ratio both decreased, reaching minimal levels around the fifth day, and then increased at 10 days after the treatment. The difference of PS value between the treatment group and the control group was statistically significant $(p<0.05)$, but there was no significant difference between the 10 th day and the 5 th day $(p=0.69) ; T / N$ values were statistically significant in each group $(p<0.01)$. Compared with the control group, the blood flow (BF) value of the rh-endostatin group first increased and then decreased (the highest point appeared at the 5th day), and was significantly higher $(p<0.01)$. There were no significant changes in other parameters of perfusion, including blood volume (BV) and mean transit time (MTT). These results indicate that improvements of blood perfusion and hypoxic condition as a result of rh-endostatin treatment appeared approximately 7 days after the treatment.

In summary, rh-endostatin endostatin can remodel and normalize tumor blood vessels, which can significantly improve the hypoxic condition, increase the concentration of the drug inside tumor tissues, and thus increase the benefit of the combined anti-cancer agents. This may be the underlining molecular mechanism supporting the combination of pretreatment with rh-endostatin with subsequent radiotherapy, chemotherapy or biological therapy. More prospective clinical studies are warranted to further optimize rh-endostatin pre-treatment in human cancers.

\section{EXPLORATION OF PREDICTIVE BIOMARKERS/EVALUATION SYSTEM FOR RH-ENDOSTATIN}

Clinical studies have shown that not all patients benefit from anti-angiogenic agents. These agents target blood vessels rather than tumors directly. Therefore, tumor sizes in responsive patients do not shrink immediately. As a result, 
clinical criteria of WHO and RECIST (response evaluation criteria in solid tumors), both of which determine whether to continue or withdraw a treatment based on tumor volume change from image analysis, are not reliable and timely measures for guiding anti-angiogenesis therapy. Identification of biomarkers that predict responsiveness and help select patients who are more likely to respond to anti-angiogenesis therapy is a critical clinical issue.

\section{CT Perfusion Imaging System}

Several studies have evaluated the predictive value of the CT perfusion imaging system. A study [71] of stage IV lung adenocarcinoma showed that PFS was significantly longer in patients with a decreased permeability or BV 5 days after the administration of rh-endostatin. There was an insufficient sample size to evaluate the predictive value of the CT perfusion imaging system in the combination therapy of rhendostatin and chemotherapy. Wu et al. [72] explored CT perfusion imaging in osteosarcoma anti-angiogenic therapy and chemotherapy to determine the value of the application. The results showed that $\mathrm{BF}$ and $\mathrm{BV}$ decreased significantly $(p<0.05)$ after combination therapy of chemotherapy and rh-endostatin, but not in the chemotherapy alone group. There was no significant difference in tumor volume between the two groups before and after treatment $(p>0.05)$. The two-year survival rates of the combination therapy group were significantly higher than that of the chemotherapy alone group $(84.71 \%$ vs. $55.25 \%, p<0.05)$.

Later, Li et al. [73] enrolled 85 patients with locally advanced or metastatic NSCLC who underwent NP chemotherapy or combined with rh-endostatin in their study. BF, BV, MTT and PS were collected through CT perfusion imaging before and after treatment. The results showed that the BV values were significantly lower after treatment $(p=0.026)$ in patients responsive to the combination therapy. The $\mathrm{BV}$ values were also negatively correlated with the tumor progression time $(p=0.018)$ in the combination group. In addition to short term responses, long-term efficacy observations also found that, after treatment, BV and TTP were negatively correlated $(r=-0.445, p=0.018)$, indicating that long-term, stable reduction of BV may be a good indication of long-term reduction of the tumor. Subgroup analysis showed no significant difference in TTP between the SD and PR groups divided based on traditional RECIST tumor size criteria, suggesting that, compared with traditional RECIST criteria, BV change was more reliable.

Therefore, because CT perfusion imaging can simultaneously detect tumor morphology and tumor vascular function, it can more accurately reflect the curative effect of anti-angiogenic drugs, and may be used as a predictive biomarker for therapeutic responsiveness, compared with the traditional solid tumor evaluation standard.

\section{Cavity Formation in Imaging Analysis}

Cavity formation may be another useful evaluation tool for therapeutic responsiveness for rhendostatin. Abnormal proliferation of tumor cells and increased internal vascular pressure can lead to abnormal vascular structure, low or lack of blood perfusion and nutrients and thus formation of necrosis and cavities. Anti-angiogenic drugs can further inhibit blood perfusion with their anti-angiogenic effects. Crabb et al. [74] found that 8 of 33 of the patients treated with chemotherapy plus VEGFR inhibitors showed cavities (diameter $>10 \%$ of lesion diameter) compared to no cavity formation in the 18 patients with chemotherapy alone, suggesting that cavity formation may be a characteristic imaging feature for anti-angiogenic treatment. Due to the prevalence of cavity formation in patients treated with anti-angiogenic treatments, the tumor effective diameter (tumor diameter-cavity diameter) may be a more appropriate measure than the traditional RECIST standard in making accurate estimates of tumor volume.

$\mathrm{Li}$ et al. investigated cavity formation and its relationship with therapeutic responsiveness in patients with advanced NSCLC after combination therapy of rh-endostatin and NP chemotherapy [73]. Eleven out of 105 patients 
developed cavities in the tumor lesions. Three of the 11 cases were of the central type and none exhibited hemoptysis. No cavity formation was observed in the 98 patients treated with chemotherapy alone. The RR was $27.3 \%$ $(35 / 94)$ and $37.2 \%$ (3/11), respectively, for patients with or without cavities in their tumors. When the Crabb's tumor effective diameter method was used in the analysis, the RR was $100 \%(11 / 11)$. The median OS for patients with cavity formation in their tumors was longer than for patients without cavities (13.6 vs. 11.8 months, $p=0.011$ ). These results indicated that cavity formation in imaging analysis may be a unique feature associated with anti-angiogenic treatment, and may not have direct association with hemoptysis, squamous subtype or tumor location.

\section{Circulating Endothelial Cells (CEC)}

Peripheral VEGF and bFGF were found to not be associated with response to anti-angiogenic therapies [75]. This may be due to degradation of these factors or interference from a variety of other anti-angiogenic factors. As a result, researchers are increasingly turning their attention to the role of their target cells-vascular endothelial cells (endothelial cells). In the 1970s, Bouvier et al. [76] first identified CECs, whose quantitative changes reflected the degree of vascular endothelial damage. CECs are usually rare in the blood of healthy people, but increase in patients with acute inflammation and tumors.

Activation by TAFs is usually needed for CECs to become aCECs, which possess the functions of chemotaxis, adhesion and angiogenesis. aCECs are downstream of the signal transduction pathway of VEGF, and their changes may more directly reflect the relationship and balance between pro-angiogenic and anti-angiogenic factors. Mancuso et al. [77] reported a higher number of aCECs in peripheral blood lymphoma and breast cancer patients compared with healthy people, and more importantly, their aCECs levels decreased after the lymphoma patients achieved complete remission after chemotherapies, or after complete mastectomy in breast cancer patients.
In the above-described study by Li et al. [72], it was observed that aCECs in the lesion were associated with a greater median survival time $(244.4 / 105$ vs. $23.3 / 105, p=0.000)$, indicating that aCECs may be a good predictor of antiangiogenic therapeutic efficacy.

The later study by Li et al. [78] included a total of 85 patients with locally advanced or metastatic NSCLC who underwent NP chemotherapy, alone or combined with rh-endostatin. In the chemotherapy group, the aCECs values were negatively correlated with the tumor progression time $(p=0.036)$ in the combined treatment group.

A retrospective study also suggested that [79] CECs, together with serum cytokeratins (CK) levels, demonstrate predictive value in the combination therapy of rh-endostatin and paclitaxel/carboplatin, with high base level CECs and a decrease in CECs after treatment, correlating with better tumor response and longer survival.

\section{VEGFR2/KDR}

The VEGFR2 or KDR gene is a known endothelial cell target that is also expressed in NSCLC tumor cells. Results reported by M. D. Anderson Cancer Center [80] showed that increased gene copy number of KDR gene in NSCLC patients treated with adjuvant chemotherapy was associated with chemotherapy resistance, increased neovascularization, increased HIF- $1 \alpha$ level and shorter survival. The KDR gene can also be used as a biomarker for identifying patients with a high risk of relapse after adjuvant therapy who are more likely benefit from anti-VEGFR2 therapy. Studies have also demonstrated that VEGFR2 expression in tumor cells correlated with resistance to radiation therapy, and that blocking the VEGFR2 pathway may enhance the sensitivity of the tumor cells to radiation therapy.

Jiang et al. reported that patients with lung cancer brain metastases with high levels of tumor VEGFR protein or KDR gene copy number benefited more significantly from combination therapy of rh-endostatin and radiotherapy, suggesting that tumor VEGFR 
expression level or KDR gene copy number may be an effective biomarker in selection of patients for the combination therapy of rh-endostatin and radiation therapy. However, this preliminary finding will need to be further evaluated with more clinical studies.

Traditional therapeutic evaluation criteria are incapable of dynamically monitoring the efficacy of anti-angiogenic agents. The relatively low cost and clinically convenient measurement of peripheral blood CECs are of scientific and clinical application value. It is believed that with the development and standardization of detection technology in the future, CECs have the potential to become a good marker to monitor patient response, to help guide the best dosage, or to identify the most suitable patients. Peripheral blood levels of VEGF and other factors may have prognostic value. But their predictive value still remains to be further studied. The non-invasive CT perfusion imaging system is more acceptable clinically with its ability in monitoring tumor blood supply in a timely manner. Other potential technologies, such as dynamic contrast-enhanced magnetic resonance imaging (DCE-MRI), have also been explored [13]. Angiogenesis is a very complex process affected by many factors, and one biomarker may not be sufficient to reflect the response to anti-angiogenic treatment. An efficacy evaluation system must be a system that contains a variety of factors. It is believed that with a deeper understanding of tumor angiogenesis and advances in technologies, a standardized evaluation system for anti-angiogenic treatment will soon emerge.

\section{SAFETY MANAGEMENT}

\section{Cardiotoxicity}

The primary toxicities of rh-endostatin are cardiac toxicity, including myocardial ischemia, mild ST-T change detected by ECG, atrioventricular block, and atrial or ventricular premature contraction, most of which were reversible, and more common in patients with previous history of coronary heart disease or hypertension. It has been proposed that anti-angiogenic drugs may cause high blood pressure and increase peripheral vascular resistance, both of which may contribute to the development of congestive heart failure. In addition, even though there was evidence suggesting that rhendostatin had limited effects on normal tissues, such as coronary vascular endothelial cells, it may not be ruled out as another contributing factor.

Therefore, a detailed medical history-including history of congestive heart failure, highrisk arrhythmia, active cardioangina, heart valvular disease or severe myocardial infarction-should be obtained before initiation of rh-endostatin treatment. Rh-endostatin should be used with caution in these high-risk patients. Ideally, patients should have a left ventricular ejection fraction (LVEF) above 0.55 before receiving rh-endostatin treatment. Cardiac function should be monitored regularly before and during treatment for early detection and treatment of cardiac adverse events. Chemotherapeutic agents such as anthracycline should be avoided in the combination treatment. Preventative and protective use of coenzyme Q10 and fructose phosphate may be prescribed when needed.

\section{Gastrointestinal Reactions}

Gastrointestinal adverse events are rare $(<5 \%)$, and mainly present as diarrhea and liver dysfunction. These reactions are usually reversible and mild and do not require special treatment. Moderate and severe reactions can be remedied with anti-symptomatic treatment and by decreasing the infusion rate, or temporary withdrawal, of the endostatin. Patients can usually resume the rh-endostatin treatment after their symptoms are relieved.

\section{Skin Reactions}

Rh-endostatin-induced allergic reactions mainly present as systemic rash, itchiness, fever, chest tightness, palpitation, and fatigue. Even though, in 2002, a phase II trial in the U.S. [81] reported that 37 patients (90\%) in the trial had 1 to 2 injection site skin reactions due to the 
intramuscular injection of rh-endostatin, skin allergic reactions are now rarely reported, as since that time the preferred administration route has changed to IV infusion.

\section{Adverse Effects Associated with Combination Chemotherapy}

A study by Shi et al. [82] showed that the main adverse events associated with rh-endostatin combined with chemotherapy included hematologic toxicity, gastrointestinal reactions, fatigue, hair loss, and pain. Yang et al. [83] also observed ECG changes, ST-T changes, and sinus tachycardia as the common adverse events, all of which were mild and relieved after withdrawal from the treatment. Five patients presented with serious adverse events in a study by Wang et al. [84]. Out of the 5, 3 deaths occurred in the rh-endostatin combination group, with causes of death being severe abdominal pain, and severe infection due to bone marrow suppression. Two deaths occurred in the control group, with the cause of death being severe infection and respiratory failure.

Sixty-eight patients were enrolled in a randomized, controlled, multi-institutional phase II study aiming at evaluating rh-endostatin as a single agent for advanced NSCLC [85]. All adverse reactions observed were mild (grade $1 / 2$ ) with no grade $3 / 4$ adverse reactions occurring. Analyses of several randomized controlled clinical trials also showed that the bone marrow suppression sometimes observed in the combination treatments was mainly associated with the chemotherapeutic agents, not with rh-endostatin. In addition, rh-endostatin did not increase the adverse effects of chemotherapy drugs.

A bleeding tendency sometimes observed in the combination therapy mainly presented as epistaxis (nasopharyngeal carcinoma) and bloody sputum (lung metastases), and were usually transient and alleviated with the use of hemostatic drugs.

Results from a phase III study in advanced NSCLC [85] showed that the bleeding rate was very low $(0.61 \%)$ with no grade $3 / 4$ bleeding. Similar findings were reported in a large phase IV study $(n=2725)$ presented on the 2010 ASCO annual meeting [9]. No severe hemoptysis was reported in the squamous cell carcinoma patients.

Retinal hemorrhage was occasionally reported in patients with a history of diabetes who had been treated with rh-endostatin therapies. Therefore, when treating patients with long diabetes history or with unstable blood sugar level, physicians need to be vigilant in monitoring the patient to prevent retinal hemorrhage.

In summary, whether alone or in combination with chemotherapy, the adverse events of rh-endostatin are primarily limited to the cardiovascular system and are mostly mild and reversible. With measures such as pre-screening patients and treating high-risk patients with caution, close observation of patients during treatment, and timely prescription of antisymptomatic treatments, these adverse events can be minimized. Further clinical observations of the safety and mechanism of action for this promising new medicine are warranted.

\section{CONCLUSION}

Accumulating evidence from clinical studies has demonstrated the significant survival benefit of rh-endostatin treatment for late stage NSCLC, which has resulted in the approval of the drug by CFDA. A large body of clinical data and experience has been obtained from studies using rh-endostatin to treat a variety of different cancers. On the basis of these clinical results, this review has summarized current clinical knowledge of rh-endostatin including its survival benefits, optimized dosages, routes of administration, recommended duration and frequency of treatment, predictive biomarkers, and its safety profile in lung cancers as well as other cancers. Further clinical studies of its safety, selection biomarkers, and basic research of its mechanism of action are warranted for this promising new drug. 


\section{ACKNOWLEDGEMENTS}

Funding. No funding or sponsorship was received for this study or publication of this article. The article processing charges were funded by the authors.

Authorship. All named authors meet the International Committee of Medical Journal Editors (ICMJE) criteria for authorship for this manuscript, take responsibility for the integrity of the work as a whole, and have given final approval for the version to be published.

Disclosures. Kai Li, Mingliang Shi and Shukui Qin have nothing to disclose.

Compliance with Ethics Guidelines. This article is based on previously conducted studies and does not involve any new studies of human or animal subjects performed by any of the authors.

Open Access. This article is distributed under the terms of the Creative Commons Attribution-NonCommercial 4.0 International License (http://creativecommons.org/licenses/ by-nc/4.0/), which permits any noncommercial use, distribution, and reproduction in any medium, provided you give appropriate credit to the original author(s) and the source, provide a link to the Creative Commons license, and indicate if changes were made.

\section{REFERENCES}

1. Kerbel RS. Tumor angiogenesis. N Engl J Med. 2008;358(19):2039-49.

2. O'Reilly MS, Boehm T, Shing Y, Fukar N, Vasios G, Lane WS, et al. Endostatin: an endogenous inhibitor of angiogenesis and tumor growth. Cell. 1997;88(2):277-85.

3. Fu Y, Tang H, Huang Y, et al. Unraveling the mysteries of endostatin. IUBMB Life. 2009;61(6): 613-26.

4. Limaverde-Sousa G, Sternberg C, Ferreira CG. Antiangiogenesis beyond VEGF inhibition: a journey from antiangiogenic single-target to broadspectrum agents. Cancer Treat Rev. 2014;40(4): 548-57.

5. Walia A, Yang JF, Huang YH, et al. Endostatin's emerging roles in angiogenesis, lymphangiogenesis, disease, and clinical applications[J]. Biochim Biophys Acta. 2015;1850(12):2422-38.

6. Boehm T, Folkman J, Browder T, et al. Antiangiogenic therapy of experimental cancer does not induce acquired drug resistance. Nature. 1997; 390(6658):404-7.

7. Fukumoto S, Morifuji M, Katakura Y, Nakamura S. Endostatin inhibits lymph node metastasis by a down-regulation of the vascular endothelial growth factor C expression in tumor cells. Clin Exp Metastasis. 2005;22(1):31-8.

8. Liu BD, Zhi XY. Evaluation of local curative effect of image-guided thermal ablation in the treatment of lung. Chin J Front Med Sci. 2015;2:11-4.

9. Sun Y, Wang JW, Liu YY, Yu QT, Zhang YP, Li K, et al. Long term results of a randomized, doubleblind, and placebo controlled phase III trial: Endostar (rh-endostatin) versus placebo in combination with vinorelbine and cisplatin in advanced nonsmall cell lung cancer. Thorac Cancer. 2013;4(4):440-8.

10. Wang J, Sun Y, Qin S, Endostar Phase IV Study Group. Results of phase IV clinical trial of combining endostar with chemotherapy for treatment of advanced non-small cell lung cancer (NSCLC). J Clin Oncol. 2010;28(suppl; abstr 7598):15s.

11. Han B, Xiu Q, Wang H, Shen J, Gu AQ, Luo Y, et al. A multicenter, randomized, double-blind, placebocontrolled study to evaluate the efficacy of paclitaxel-carboplatin alone or with endostar for advanced non-small cell lung cancer. J Thorac Oncol. 2011;6(6):1104-9.

12. Wang J, Li K, Sun T, Zhang MJ, Li WL, Yao Q, et al. The efficacy and safety of docetaxel combined with recombinant human endostatin in the treatment of non-small cell lung cancer with or without intolerance after treatment. Chin J Oncol. 2013;35(8):618-22.

13. Jiang XD, Ding MH, Qiao Y, Liu Y, Liu L. Study on lung cancer cells expressing VEGFR2 and the impact on the effect of RHES combined with radiotherapy in the treatment of brain metastases. Clin Lung Cancer. 2014;15(2):e23-9.

14. Zhang R, Wang ZY, Li YH, Lu YH, Wang S, Yu WX, et al. Usefulness of dynamic contrast-enhanced magnetic resonance imaging for predicting treatment response to vinorelbine-cisplatin with or 
without recombinant human endostatin in bone metastasis of non-small cell lung cancer. Am J Cancer Res. 2016;6(12):2890-900.

15. Jiang XD, Dai P, Wu J, Song DA, Yu JM. Effect of recombinant human endostatin on radiosensitivity in patients with non-small-cell lung cancer. Int J Radiat Oncol Biol Phys. 2012;83(4):1272-7.

16. Bao Y, Peng F, Zhou QC, Yu ZH, Li JC, Cheng ZB, et al. Phase II trial of recombinant human endostatin in combination with concurrent chemoradiotherapy in patients with stage III non-small-cell lung cancer. Radiother Oncol. 2015;114(2):161-6.

17. Ma HL, Hui ZG, Zhao LJ, Xu YJ, Zhai YR, Wu RY, et al. Treatment of non-resectable stage III NSCLC with continuous intravenous injection of Endostar: results from a phase II prospective multicenter clinical trial results. Chin J Radiat Oncol. 2016;25(2):114-8.

18. Yang YZ, Pan LK, Qi DL, Xin L, Cui Y, An GY, et al. Effect of recombinant human endostatin combined with TP regimen on postoperative adjuvant therapy for non-small cell lung cancer. Med J Chin Lib Army. 2012;37(1):49-53.

19. Zhao J, Wang W, Shang LQ, Xiao W, Li XC, Song WA, et al. Comparison of postoperative adjuvant chemotherapy with combined adjuvant chemotherapy and non-small cell lung cancer. Transl Med J. 2014;3(1):19-21.

20. Pan F, Gu AQ, Wang WM, Jiang LY. Effect of recombinant human endostatin combined with platinum-based postoperative adjuvant chemotherapy on non-small cell lung cancer. World Clin Drugs. 2015;5:322-6.

21. Zhou ZT, Zhou FX, Wei Q, Zou LY, Qin BF, Peng XS. Phase II study of cisplatin/etoposide and endostar for extensive-stage small-cell lung cancer. Cancer Chemother Pharmacol. 2011;68(4):1027-32.

22. Lu S, Li L, Luo Y, Zhang L, Wu G, Chen ZW, et al. A multicenter, open-label, randomized phase II controlled study of rh-endostatin (Endostar) in combination with chemotherapy in previously untreated extensive-stage small-cell lung cancer. J Thorac Oncol. 2015;10(1):206-11.

23. Chen JH, Luo YZ, Zhou WW, Zhou H, Wang W. Clinical observation of recombinant human endostatin combined with carboplatin and etoposide in the treatment of advanced small cell lung cancer. J Clin Med Pract. 2013;17(5):26-8.

24. Qin SK, Yang LQ, Liang J, Cheng Y, Tan QH, Bi JW, et al. Intraventricular application of recombinant human endostatin and/or cisplatin in the treatment of malignant pleural effusion of the prospective, randomized, national multi-center clinical study. Chin Clin Oncol. 2017;22(3):193-202.

25. Zhou JF, Bai CM, Wang YZ, et al. Endostar combined with chemotherapy for treatment of metastatic colorectal and gastric cancer: a pilot study [J]. Chin Med J (Engl). 2011;124(24):4299-303.

26. Xu HX, Huang XE, Qian ZY, Xu X, Li Y, Li CG. Clinical observation of Endostar ${ }^{\circledR}$ combined with chemotherapy in advanced colorectal cancer patients. Asian Pac J Cancer Prev. 2011;12(11):3087-90.

27. Li BL, Hu XL, Zhao XH, Sun HG, Zhou CY, Zhang Y. Endostar combined with irinotecan/calcium folinate/5-fluorouracil (FOLFIRI) for treating advanced colorectal cancer: a clinical study. J Chemother. 2015;27(5):301-6.

28. Chen Z, Guo W, Cao J, Lv F, Zhang W, Qiu L, et al. Endostar in combination with modified FOLFOX6 as an initial therapy in advanced colorectal cancer patients: a phase I clinical trial. Cancer Chemother Pharmacol. 2015;75(3):547-57.

29. Pan YQ, Jiao GL. A meta-analysis of the short-term efficacy of degrading combined with chemotherapy in the treatment of advanced colorectal. J South Med Univ. 2014;2:270-4.

30. Qi XY, Yu ZY, Ouyang XN, Wang WW. Clinical observation of recombinant human vascular endostatin combined with chemotherapy in the treatment of advanced metastatic colorectal cancer. J Mod Oncol. 2015;17:2459-63.

31. Guo YB, Zhen L, Zhu WR, Xu X, Li Y, Li CG. Clinical observation of recombinant human endostatin combined with chemotherapy in the treatment of metastatic colorectal cancer. Chin Clin Oncol. 2011;16(8):742-5.

32. Cui C, Mao L, Chi Z, Si L, Sheng XN, Kong Y, et al. A phase II, randomized, double-blind, placebo-controlled multicenter trial of Endostar in patients with metastatic melanoma. Mol Ther. 2013;21(7):1456-63.

33. Cui C, Si L, Chi Z, Sheng XN, Mao LL, Wang X, et al. Preliminary results of a phase II trial with continuous intravenous infusion of rh-endostatin in combination with dacarbazine as the first-line therapy for metastatic acral melanoma. J Clin Oncol. 2015 (suppl; abstre20087).

34. Xu R, Ma N, Wang F, Ma L, Chen R, Chen R, et al. Results of a randomized and controlled clinical trial evaluating the efficacy and safety of combination therapy with Endostar and S-1 combined with oxaliplatin in advanced gastric cancer. Onco Targets Ther. 2013;6:925-9. 
35. Qu XL, Chai NX, Wang YJ, Han Y, Zhang Y, Tao L. Recombinant human endostatin combined with DCF regimen for neoadjuvant chemotherapy patients with advanced gastric cancer to improve the rate of radical surgery. Tumor. 2011;31(8):765-7.

36. Su H, Hu B, Ji CS, He YF, Han XH, Lv YJ. Meta analysis of recombinant human endostatin combined with chemotherapy in the treatment of advanced gastric cancer. Tumor. 2015;35(3):322-32.

37. Jin T, Li B, Chen XZ. A phase II trial of Endostar combined with gemcitabine and cisplatin chemotherapy in patients with metastatic nasopharyngeal carcinoma (NCT01612286). Oncol Res. 2013;21(6):317-23.

38. Guan Y, Li A, Xiao W, Liu S, Chen B, Lu T, et al. The efficacy and safety of Endostar combined with chemoradiotherapy for patients with advanced, locally recurrent nasopharyngeal carcinoma. Oncotarget. 2015;6(32):33926-34.

39. Du HJ. Clinical application of recombinant human endostatin combined with transcatheter arterial chemoembolization in the treatment of advanced liver cancer. J Interv Radiol. 2009;18(4):302-5.

40. Su Y, Zhou ZH, Wang Q. Efficacy and safety of recombinant human endostatin in the treatment of advanced liver cancer by transcatheter arterial chemoembolization. J Pract Hepatol. 2014;6:644-6.

41. Lin Q, Zhang SY, Huang N, Su YH. Meta-analysis of recombinant human endostatin injection combined with transcatheter arterial chemoembolization for primary hepatocellular carcinoma. World Chin J Digestol. 2015;4:655-64.

42. Jia $\mathrm{Q}, \mathrm{Xu} \mathrm{J}$, Jiang $\mathrm{W}$, et al. Dynamic contrastenhanced MR imaging in a phase II study on neoadjuvant chemotherapy combining Rh-endostatin with docetaxel and epirubicin for locally advanced breast cancer [J]. Int J Med Sci. 2013;10(2):110-18.

43. Lu Y, Huang HX, Li GS. Clinical study of endostatin combined with GP regimen in the treatment of metastatic triple-negative breast cancer. Chin J Clin Oncol. 2012;39(23):1946-8.

44. Huang W, Liu J, Wu F, Chen K, Li N, Hong Y, et al. The efficacy and safety of endostar combined with taxane-based regimens for HER-2-negative metastatic breast cancer patients. Oncotarget. 2016;7(21):31501-7.

45. Zhong Z, Gu X, Zhang Z, Wang D, Qing Y, Li M, et al. Recombinant human endostatin combined with definitive chemoradiotherapy as primary treatment for patients with unresectable but without systemic metastatic squamous cell carcinoma of the oesophagus. Br J Radiol. 1019;2012(85):e1104-9.
46. Lv JH, Li T, Deng XR, Li F, Song YQ, Li CR, et al. Randomized phase II study of recombinant human endostatin combined with definitive chemoradiotherapy in locally advanced esophageal squamous cell carcinoma. J Clin Oncol 2015. (suppl; abstr 4035)

47. Ren TJ, Shan FX, Hou JF, Xue Q. Clinical observation of XELOX regimen combined with Endostar in the treatment of advanced biliary cancers. Chin J Oncol Prev Treat. 2015;7(1):41-4.

48. Li R, Sk Qin, Liu XF, Gong XL, Hua HQ, Wang L, et al. Preliminary observation of GEMOX regimen combined with recombinant human endostatin in the treatment of advanced biliary cancers. Chin Clin Oncol. 2014;5:430-4.

49. Su A, Zhang J, Pan ZH, Zhou QM, Lv X. Salvage therapy of gemcitabine plus endostar significantly improves progression-free survival (PFS) with platinum-resistant recurrent epithelial ovarian cancer. Asian Pac J Cancer Prev. 2013;14(3):1841-6.

50. Zhang C, Deng WY, Li N, Xu YF, Zhang YP, Wei C, et al. Clinical observation on treatment of advanced ovarian cancer with different routes of administration. Cancer Res Prev Treat. 2016;43(1):54-7.

51. Ke QH, ZhouSQ HuY, Liu Z, Zhang WT, Su XY, et al. Short-term efficacy of recombinant human endostatin (Endu) combined with radiotherapy in the treatment of 48 cases of advanced cervical cancer. J Mod Oncol. 2011;19(10):2066-8.

52. Ke QH, Zhou SH, Du F, Huang M, Luo F, Lei Y, et al. Simultaneous radiotherapy and chemotherapy combined with recombinant human endostatin in the treatment of 52 cases of advanced cervical cancer. Pract J Cancer. 2012;27(4):373-5.

53. Nong XS, Huang XS, Mo YF, Liang D. Short-term efficacy of Endostar combined with gemcitabine in the treatment of advanced cervical cancer. J Guangxi Med Univ. 2015;32(4):646-8.

54. Xu M, Xu CX, Bi WZ, Song ZG, Jia JP, Chai W. Effects of endostar combined multidrug chemotherapy in osteosarcoma. Bone. 2013;57(1):111-5.

55. Xu HR, Li B, Huang Z, Zhang Q, Niu XH. A prospective, concurrent controlled non - randomized clinical study of chemotherapy combined with recombinant human endostatin in the treatment of stage IIB osteosarcoma. Chin Clin Oncol. 2013;18(5):421-5.

56. Yan HL, Zhang ZC, Yang QC, Qin S, Dong Y. A prospective, non-randomized clinical study of recombinant human endostatin combined with chemotherapy in the treatment of osteosarcoma. Prog Mod Biomed. 2015;15(25):4866-71. 
57. Zhang LP, Liao XY, Xu YM, Yan LJ, Yan GF, Wang $\mathrm{XX}$, et al. Efficacy and safety of endostar combined with chemotherapy in patients with advanced soft tissue sarcomas. Asian Pac J Cancer Prev. 2013;14(7):4255-9.

58. Huang XF, Qu Z, Hu W, Zhang N, Wang YF, Wang $\mathrm{Y}$, et al. Treatment of metastatic soft tissue sarcoma with recombinant human endostatin combined with chemotherapy Bed observation. J Mod Oncol. 2015;23:3489-92.

59. Xing P, Zhang J, Yan Z, Zhao G, Li X, Wang G, et al. Recombined humanized endostatin (Endostar) combined with chemotherapy for advanced bone and soft tissue sarcomas in stage IV. Oncotarget. 2016. https://doi.org/10.18632/oncotarget.13545.

60. Li Y, Zhang JP, Wang ZW, Zhang XZ, Zhou WK. Clinical study of recombinant human vascular endothelin combined with temozolomide in the treatment of recurrent high-grade gliomas. Chin J Brain Dis Rehabil (Electron Ed). 2014;2:8-11.

61. Zhang JP, Teng Y, Li C. Recombinant human endostatin combined with cytotoxic drugs to rescue chemotherapy for recurrence of glioblastoma. Chin J New Drugs Rem. 2015;4:310-4.

62. Li N, Jin ZL, Liu ZJ, Wang J, Li K. Recombinant human endostatin injection combined with chemotherapy for multi-cycle treatment of advanced non-small cell lung cancer. Chin J Oncol. 2011;33(12):937-42.

63. Hu W, Fang J, Nie J, Dai L, Zhang J, Chen XL, et al. Efficacy and safety of extended use of platinumbased doublet chemotherapy plus endostatin in patients with advanced non small cell lung cancer. Medicine (Baltimore). 2016;95(28):e4183.

64. Hansma AH, Broxterman HJ, van der Horst I, Yuana Y, Boven E, Giaccone G, et al. Recombinant human endostatin administered as a 28-day continuous intravenous infusion, followed by daily subcutaneous injections: a phase I and pharmacokinetic study in patients with advanced cancer. Ann Oncol. 2005;16(10):1695-701.

65. Huang Y, Yang Y, Zhao H, Ma YX, Zou QF, Zhang L. Phase I study of continuous intravenous infusion of rh-endostatin combined with pemetrexed and carboplatin in advanced NSCLC. J Thorac Oncol. 2015;10(9):S2-315.

66. Ning T, Yan X, Lu ZJ, Wang GP, Zhang NG, Yang JL, et al. Gene therapy with the angiogenesis inhibitor endostatin in an orthotopic lung cancer murine model. Hum Gene Ther. 2009;20(2):103-11.

67. Huang G, Chen L. Recombinant human endostatin improves anti-tumor efficacy of paclitaxel by normalizing tumor vasculature in Lewis lung carcinoma. J Cancer Res Clin Oncol. 2010;136(8):1201-11.

68. Li N, Zheng D, Wei X, Jin Z, Zhang C, Li K. Effects of recombinant human endostatin and its synergy with cisplatin on circulating endothelial cells and tumor vascular normalization in A549 xenograft murine model. J Cancer Res Clin Oncol. 2012;138(7):1131-44.

69. Peng F, Xu Z, Wang J, Chen YY, Li Q, Zuo YF, et al. Recombinant human endostatin normalizes tumor vasculature and enhances radiation response in xenografted human nasopharyngeal carcinoma models. PLoS One. 2012;7(4):e34646.

70. Jiang XD, Dai P, Qiao Y, Wu J, Song DA, Li SQ. Clinical study on the recombinant human endostatin regarding improving the blood perfusion and hypoxia of non-small-cell lung cancer. Clin Transl Oncol. 2012;14(6):437-43.

71. Huang MJ, Yu M, Zhang K, Li YY, Liu HM, Li WJ, et al. Early CT perfusion changes and the outcome of antiangiogenic therapy and chemotherapy in patients with advanced primary lung adenocarcinoma. J Clin Oncol. 2016;34(suppl; abstr e20543).

72. Wu CM, Ji JY, Shi X, Wu M, Deng G. Application of CT perfusion imaging in evaluation of therapeutic effect of osteosarcoma. Chin J Med Imaging Technol. 2011;27(6):1272-5.

73. Huang C, Wang X, Wang J, Lin L, Liu ZJ, Xu WJ, et al. Incidence and clinical implication of tumor cavitation in patients with advanced non-small cell lung cancer induced by Endostar, an angiogenesis inhibitor. Thorac Cancer. 2014;5(5):438-46.

74. Crabb SJ, Patsios D, Sauerbrei E, Ellis PM, Arnold A, Goss G, et al. Tumor cavitation: impact on objective response evaluation in trials of angiogenesis inhibitors in non-small-cell lung cancer. J Clin Oncol. 2009;27(3):404-10.

75. Zhang CC, Wang J, Li K. Evaluation of the efficacy of anti-angiogenesis: how far is the new standard? Chin Clin Oncol. 2011;10:951-5.

76. Bouvier CA, Gaynor E, Cintron JR, Bernhardt B, Spaet TH. Circulating endothelium as an indication of vascular injury. Thromb Diath Haemorrh. 1970;40(Suppl.):163-8.

77. Mancuso P, Bertolini F. Circulating endothelial cells as biomarkers in clinicaloncology. Microvasc Res. 2010;79(3):224-8.

78. Wang J, Xiao J, Wei X, Wang L, Lin L, Liu Z, et al. Circulating endothelial cells and tumor blood 
volume as predictors in lung cancer. Cancer Sci. 2013;104(4):445-52.

79. Chu TQ, Ding H, Garfield DH, Gu AQ, Pei J, Du $\mathrm{WD}$, et al. Can determination of circulating endothelial cells and serum caspase-cleaved CK18 predict for response and survival in patients with advanced non-small-cell lung cancer receiving endostatin and paclitaxel-carboplatin chemotherapy? a retrospective study. J Thorac Oncol. 2012;7(12):1781-9.

80. Yang F, Tang X, Riquelme E, Behrens C, Nilsson $\mathrm{MB}$, Giri $\mathrm{U}$, et al. Increased VEGFR-2 gene copy is associated with chemoresistance and shorter survival in patients with non-small-cell lung carcinoma who receive adjuvant chemotherapy. Cancer Res. 2011;71(16):5512-21.

81. Twombly R. First clinical trials of endostatin yield lukewarm results. J Natl Cancer Inst. 2002;94(20):1520-1.
82. Shi HL, Xu LY, Liu Z. Clinical study of recombinant human endostatin (YH-16) injection in the treatment of advanced non-small cell lung cancer. Chin J Lung Cancer. 2004;7(4):325-8.

83. Yang L, Wang JW, Tang ZM, Liu XW, Huang J, Li ST, et al. Phase I Clinical study of recombinant human endostatin. Chin J New Drugs. 2004;13(6):548-53.

84. Wang WJ, Sun Y, Liu Y, Yu QT, Zhang YP, Li K, et al. Recombinant human endostatin combined with NP regimen for the treatment of advanced NSCLC in a randomized, double-blind, controlled, multicenter phase III clinical study. Chin J Lung Cancer. 2005;8(4):283-90.

85. Yang L, Wang JW, Sun Y, Zhu YZ, Liu XQ, Li WL, et al. Randomized phase II trial on escalated doses of Rh-endostatin (YH-16) for advanced non-small cell lung cancer[J]. Chin J Oncol. 2006;28(2):138-41. 